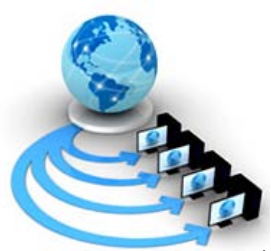

Volume 9, No. 3, May-June 2018

\title{
ANALYSIS OF COLLABORATIVE FILTERING WITH I-SSU RECOMMENDATION SYSTEM
}

\author{
E.Kaviyavarshini \\ Part time Research Scholar \\ Department of Computer Science(P.G) \\ Kongu Arts and Science College(Autonomous) \\ Erode, TamilNadu, India
}

\author{
P.Kalarani \\ Assistant Professor \\ Department of Computer Technology and \\ Information Technology \\ Kongu Arts and Science College(Autonomous) \\ Erode, Tamilnadu, India
}

\begin{abstract}
Social recommendation is common and prosperous among varied urban property applications like merchandise recommendation, online sharing, and looking services. Users build use of those applications to create many implicit social networks through their daily social interactions. The users in such social networks will rate some fascinating things and provides comments. The bulk of the present studies investigates the rating prediction and recommendation of things supported user-item bipartite graph and user-user social graph, therefore, referred to as social recommendation. However, the spacial issue wasn't thought-about in their recommendation mechanisms. With the speedy development of the service of location-based social networks, the spacial info step by step affects the standard and the correlation of rating and recommendation of things. This paper survey a location-based social network (LBSN) doesn't solely mean adding a location to Associate in Nursing existing social network so individuals within the social organization will share location-embedded info, however conjointly consists of the new social organization created from people connected by the interdependence derived from there a panorama of the recommendations locations within the physical world still as their location-tagged media content, like photos, videos and text. This paper surveys on locationbased social networks with a balanced depth, facilitating analysis into this rising topic.
\end{abstract}

Keywords: Social Recommendation, Privacy Policy Mining, Location Based Social Networking, Recommendation, SSU, i-SSU

\section{INTRODUCTION}

Social network communication is often a stand to make group relations among user that distribute parallel importance, activities, backgrounds, or real-life connections. A social network service consists of an illustration of each user sometimes a profile, his or her social links, and a selection of additional services. Social network sites unit of measurement web-based services that let folks create a public profile, turn out a listing of users with whom to share and examine the connections. Most social network services unit of measurement web-based and provide suggests that for users to act over cyberspace, like email and instant electronic communication. Social network sites unit of measurement varied which they incorporate new data and communication tools like mobile property, photo, video sharing. The net community services unit of measurement usually thoughtabout a social network service, although in an exceedingly very broad sense, the social network service usually suggests that academic degree individual-centered service whereas online community services unit of measurement group-centered. Social media allow users to share ideas, pictures, posts, activities, events, and interests of people in their network.

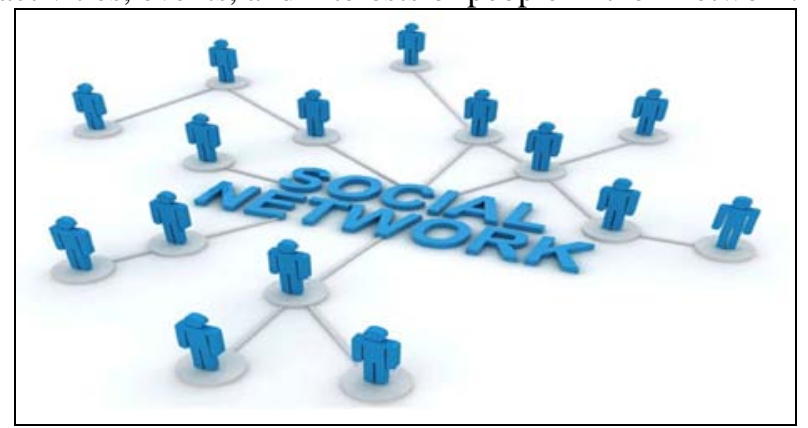

Fig 1.1 Social Networks Model

\section{A. Uses of Social Group Activity}

Social Group activity square measures the favored trend in trendy days. With its vast quality, little business homes have additionally started mistreatment social networking websites for complete promotion. Today's age is AN age of advanced technology. With the boon of web reaching nearly every corner of the globe, there has been a vast transformation in each and every field. Be it putting in place a stronger platform of communication or connecting the world beneath a standard network, the web has actually contributed in creating world abundant a smaller place to measure it.

From video chats to Video conferencing, from on-line promoting to socializing via social media, the web has actually and for sure a blessing for the worldwide societies. Social media promoting is (SMM) observed outline bound websites that facilitate interpersonal communication through bound websites wherever in individuals will produce their own profile page and communicate with friends and associates through on-line messages or scraps. A user will produce a network of friends, produce a bunch, initiate or participate in a very word. This Social communication model became a tool that made-up the manner for the advanced mode of communication between all the networks and web users.

The social communication model not solely remained a stage to initiate informal dialogues and a supporter of live messages, however, became an integral a part of promoting ways of the many business homes. The appliance of those sites has unfolded to business homes that started mistreatment the Social Networking sites as a platform to push their services and build complete awareness. The enterprises started to practice these on-line communities or websites for developing contacts and driving traffic to their individual websites. These social networking websites find the foremost tool of social 
media promoting. The foremost ordinarily used websites Twitter and Face book unit Social Networking Sites that help friends and colleagues to share dialogues with each other through Wall Posts, Messages, and Comments.

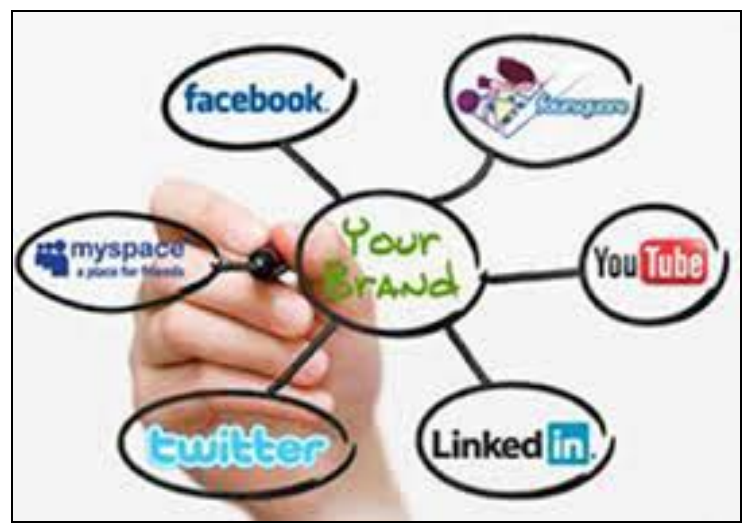

Fig 1.2 Social Communication Model

\section{RELATED WORKS}

\section{A. Location-Based Social Networks}

Newly, move forward place- attainment and wireless communication technologies have been enabled the creation of location-based social networking services. The service provides a user will simply share their geospatial scene related services among the physical world via online platforms. let's say, a user with a movable will share comments beside his social network one or two of the buildings at that he has dined on an online social site. As an associate degree example, those that constantly hike on the identical mountain are going to be placed in contact.

* The position facet bridges the gap between the physical world and also the digital on-line social networking services, giving rise to new opportunities and challenges in ancient recommender systems within the following aspects:

* Complex objects and relations: A location could be a new object in location-based social networks (LBSNs), generating new relations between users, between locations, and between users and locations. New recommendation eventualities, like location and itinerary recommendations, may be enabled mistreatment this new information, and ancient recommendation eventualities, like friend and media recommendation, may be increased. However, doing, therefore, needs new methodologies for generating high-quality recommendations.

* Rich knowledge: A location is one in all the foremost vital parts process a user's context. In-depth information, a couple of user's behavior and preferences may be learned via their location history. The large volume of location-related knowledge generated by users improves the probability that social opinions, e.g., the foremost favorite dish in an exceedingly building or the foremost fashionable activity at a degree of interest, may be accurately assessed by counseled systems.
These projections are tackled by several new moves towards to recommendation systems, victimization totally (different|completely different) knowledge sources and methodologies to get different forms of recommendations. It's projected to supply a replacement system and analyze 1) what a made recommendation is (i.e., the target of a recommendation), 2) the methodology used to get a recommendation, and 3) the info supply it used. In step with these three aspects, we tend to propose three taxonomies to severally partitions the recommender systems. The contributions of this thesis square measure careful as follows:

* To distinguish LBSNs from standard social networks and outline their distinctive properties, challenges, and opportunities.

* For the type of the key recommended model for LBSNs in few taxonomies, organized by knowledge sources, methodologies, and recommendation objectives model.

* In every class, we tend to summarize the goals and contributions of every system. Additionally, our approach highlights one representational system in every class, providing a lot of in-depth read of the methodology.

* To summarize the key ways for evaluating the recommendations in LBSN.

* To signify capable analysis directions in LBSN reference systems, paying special attention to the directions that result from the analysis and synthesis of the various suggestion system classes.

\section{LITERATURE SURVEY}

Zan Huang, Daniel Zeng and Hsinchun Chen [1] express a collaborative filtering is one of the a large amount widely adopted and successful recommendation approaches. The proposed model based the natural properties of the $\mathrm{CF}$ framework. The problem definition of CF model is a human interaction design and analysis. However the available data about item interaction, social application. The CF model applies multi grade rating data to binary data and modification.

Haiqin Yang, Guang Ling, Yuxin Su, Michael R. Lyu, and Irwin King [2] describe Recommender systems are promising for providing personalized favorite services. Collaborative filtering (CF) technologies, creating a prediction of users' preference supported users' previous behaviors, became one among the foremost triple-crown techniques to create trendy recommender systems. Many difficult problems occur in antecedently projected CF methods:

* Most CF ways ignore users' response patterns and will yield biased parameter estimation and suboptimal performance;

* Some CF ways adopt heuristic weight settings, that lacks a systematical implementation;

* The multinomial mixture models could weaken the processing ability of matrix factoring for generating the info matrix, therefore increasing the process value of coaching.

To resolve these problems, incorporate users' response models into the probabilistic matrix factoring (PMF), 
a preferred matrix factoring CF model, to ascertain the Response Aware Probabilistic Matrix factoring (RAPMF) framework. Extra expressly, they create the hypothesis on the user response as a Bernoulli sharing that is parameterized by the rating scores for the determined ratings whereas as a step performed for the unobserved ratings. Also, they speed up the formula by a mini-batch execution and a crafting programming strategy. This paper concludes, several protocol model evaluations on both real datasets to improve the accuracy of RAPME model.

Chinnu Priya J.V and Suja Rani M.S [3] describe the number of web services with similar functionality increases, the service users usually depend on web recommendation systems. Nowadays the service users pay a lot of importance on non-useful properties that are called Quality of Service (QoS) whereas finding and choosing acceptable internet services. Cooperative filtering [14] approach predicts the QoS values of the net services effectively. Existing recommendation systems seldom contemplate the personalized influence of the users and services in crucial the similarity between users and services. The analysis of rating model is score, user, item and Qos based used to predict real dataset services model. The system, therefore, employs the situation info of users and repairs in choosing similar neighbors for the target user and service and thereby creating personalized service recommendations for service users.

Hsiang-Fu Yu, Cho-Jui Hsieh, Si Si, and Inderjit Dhillon [4] express a matrix factorization, when the matrix has missing values; have been one of the foremost methods for recommended systems. To handle web-scale datasets with millions of users and billions of ratings, scalability becomes an important issue. ALS and SGD methods are accepted come close to compute the matrix distribution and activity to parallelize these algorithms. However, due to the cubic time intricacy in the target rank, ALS is not scalable to large-scale datasets. The SGD carries out resourceful updates, but usually suffers from slow convergence that is sensitive to the parameters. Synchronize plunge, a classical optimization approach has been used for many other large-scale problems, but its application to matrix factorization for recommender systems has not been explored thoroughly.

It aims to design an efficient and easily parallelizable method for matrix factorization in large-scale recommender systems. It shows that coordinate descent methods are effective for nonnegative matrix factorization (NMF). This motivates us to investigate and coordinate descent approaches. They propose a coordinate descent based method, CCD++, which has fast running time and can be easily parallelized to handle data of various scales. The main contributions of this paper are:

* They proposed a scalable and efficient coordinate descent based matrix factorization method CCD++. The time complexity per iteration of CCD++ is lower than that of ALS, and it achieves faster convergence than SGD.

* They prove that CCD++ can be simply useful to problems of various scales on both shared-memory multi-core and distributed systems.
Panagiotis Symenonidis, Eleftherios Tiakas, Yannis Manolopoulos [5] are describing a social score model like user interaction and equally co-score interaction model.The more effective a part of previous add Rating Prediction and Recommendation of merchandise CF typically takes into consideration ratings of users on merchandise. However, in SRNs users may be engineered in their specific social network by adding one another as friends. A SU technique which mixes similarity matrices derived from heterogeneous (unipartite and bipartite) specific or implicit SRNs. To boot, we have a tendency to improve an efficient coefficient maneuver of SRNs influence supported their structured density. However, they failed to use a coefficient strategy to SRNs influence supported their structured information density. In this paper, we have a tendency to propose Social-Union, a way which mixes multiple similarity matrices derived from heterogeneous specific or implicit social networks. The social union model considers user profile with heterogenous communication model and observation of valuable data services.

\section{IV.METHODOLOGY}

In the social proposal, rating prediction and item reference area unit two main examine issues. Let's say, for a brand new client in E-commerce applications, a way to expeditiously predict his/her rating for a definite product and suggest some potential attention-grabbing merchandise for him/her with a social recommendation mechanism may be a challenging issue. There has been a variety of connected work [6] [12] on rating prediction and social recommendation. Recently, planned system affiliation/group recommendations supported the relationship network among users, and also the affiliation/group network between users and teams. However, their technique centered on path counts solely and failed to exploit different options and network characteristics which might be informative for link formation. In [7] [12] they planned the advising systems with the incorporation of trust and distrust data. The planned framework was supported matrix resolving with regularization terms confining the trust and distrust relations between users.

In this paper planned system for generating the location-sensitive recommendations by rating prediction of things in ad-hoc social network environments and propose abstraction social union (SSU), an associate approach that mixes numerous likeness matrices resulting from the user-item bipartite graph, user-user social graph, and user-location bipartite graph (UL-BG). SSU differs from the Social Union [8][11][12] as a result of it takes under consideration not solely the relation between user and item furthermore, because of the social relationships between users, however conjointly the relationships between user and site.

First, three varieties of similarity matrices derived from the user-item bipartite graph, user-user social graph, and user-location bipartite graph unit provided and analyzed. Second, the similarity calculation approach, an abstraction social union that mixes the three similarity matrices on is projected. Third, we have a tendency to tend to boost the FriendTNS algorithmic rule [9] [10] [12] and devise the SSUaware location-sensitive recommendation formula for things. Last, the projected SSU-aware location-sensitive 
recommendation formula is evaluated using picture Lens data set that will be an extraordinarily common picture recommendation service. The following contribution of user services model.

* Dimentional data: The data drive from user item and location based create social graph model.

* Score Analysis Model. In this method derive from User Score, Session and Age group data.

* Parallel aggregation Model. Further, It proposes associate aggregation union, specifically SSU that mixes the numerous similarity matrices simR, S(age) and simD and returns the similarity matrix between any 2 users.

* Evaluation prediction and Reference. At last, it adopts the finalized similarity matrix to predict the missing ratings and provide the recommendations in terms of similarity.

At present, social recommendations have been prospering in numerous urban property applications like on-line sharing, product recommendation and searching services [13] [15]. These applications permit users to make many implicit social networks through their daily social interactions. The users in such social networks will rate some fascinating things and provides comments. The bulk of the prevailing studies has investigated the rating prediction and recommendation of things supported user-item bipartite graph and user-user social graph, therefore, referred to as social recommendation. However, the spatial issue wasn't thought-about in their recommendation mechanisms.

Table 4.1 Algorithm Table

\begin{tabular}{|c|l|l|l|}
\hline S.No & Researcher & \multicolumn{1}{|c|}{ Algorithm } & \multicolumn{1}{c|}{ Features } \\
\hline 1 & $\begin{array}{l}\text { Panagiotis } \\
\text { Symeonidis }\end{array}$ & $\begin{array}{l}\text { SRNs } \\
\text { Recommendation } \\
\text { Algorithm }\end{array}$ & $\begin{array}{l}\text { Review Content } \\
\text { and Rating }\end{array}$ \\
\hline 2 & $\begin{array}{l}\text { Mohamed } \\
\text { Sarwat }\end{array}$ & $\begin{array}{l}\text { Dimensionality- } \\
\text { Reduction } \\
\text { Algorithm }\end{array}$ & $\begin{array}{l}\text { Dimensionality- } \\
\text { reduction }\end{array}$ \\
\hline 3 & Zan Huang & $\begin{array}{l}\text { Response Aware } \\
\text { Probabilistic } \\
\text { Matrix } \\
\text { Factorization }\end{array}$ & Rating Score \\
\hline 4 & $\begin{array}{l}\text { Haiqin } \\
\text { Yang }\end{array}$ & $\begin{array}{l}\text { QoS based service } \\
\text { ranking }\end{array}$ & $\begin{array}{l}\text { Non functional } \\
\text { properties }\end{array}$ \\
\hline 5 & Phinnu & $\begin{array}{l}\text { Alternating Least } \\
\text { Squares (ALS) } \\
\text { and Stochastic } \\
\text { Gradient Descent } \\
\text { (SGD) }\end{array}$ & $\begin{array}{l}\text { Rating and } \\
\text { Memory }\end{array}$ \\
\hline
\end{tabular}

In addition to all the prevailing system mechanism, the projected study conjointly presents people based mostly similarity measure. Here Similarity measure supported users' ages is additionally taken into the study as stone (Age) together with simR (Rating), stone (User) and simD (Location). The score prediction and references adopt/ the finalized likeness matrix with together with rock to predict the missing ratings and supply the proposals [16] additionally, the time intervals are taken for matrix calculation. The expected system has following blessings.
Only selective dataset applied user score analysis.

$>$ Session based recommendation model.

$>$ New products launched at some locations and their recommendations by the appliance itself square measure enclosed.

$>$ Age cluster-wise similarity is additionally taken into thought.

Table 4.2 Comparison Table

\begin{tabular}{|c|c|c|c|}
\hline $\begin{array}{c}\text { S. } \\
\text { No }\end{array}$ & Algorithm & Advantages & Disadvantages \\
\hline 1 & $\begin{array}{c}\text { SRNs } \\
\text { Recommendat } \\
\text { ion Algorithm }\end{array}$ & \begin{tabular}{l}
\multicolumn{2}{l}{ Similarity } \\
matrices derived \\
from \\
heterogeneous \\
explicit or \\
implicit social \\
networks
\end{tabular} & $\begin{array}{l}\text { All social } \\
\text { networks do not } \\
\text { contribute } \\
\text { equally or } \\
\text { contain valuable } \\
\text { information. }\end{array}$ \\
\hline 2 & $\begin{array}{l}\text { Dimensionalit } \\
\text { y-Reduction } \\
\text { Algorithm }\end{array}$ & $\begin{array}{l}\text { Interaction } \\
\text { matrix becomes } \\
\text { dense, and } \\
\text { computing pair- } \\
\text { wise consumer } \\
\text { similarity } \\
\text { requires } \\
\text { significant CPU } \\
\text { cycles }\end{array}$ & $\begin{array}{l}\text { Dimensionality- } \\
\text { reduction } \\
\text { algorithm } \\
\text { requires the } \\
\text { longest run time }\end{array}$ \\
\hline 3 & $\begin{array}{c}\text { Response } \\
\text { Aware } \\
\text { Probabilistic } \\
\text { Matrix } \\
\text { Factorization }\end{array}$ & $\begin{array}{l}\text { Multinomial } \\
\text { mixture models } \\
\text { may weaken the } \\
\text { computational } \\
\text { ability of } \\
\text { generating data } \\
\text { matrix and } \\
\text { increase the } \\
\text { computational } \\
\text { cost of training } \\
\text { the model. }\end{array}$ & $\begin{array}{l}\text { The rating scores } \\
\text { for the observed } \\
\text { ratings while as a } \\
\text { step function for } \\
\text { the unobserved } \\
\text { ratings. }\end{array}$ \\
\hline 4 & $\begin{array}{l}\text { QoS based } \\
\text { service } \\
\text { ranking }\end{array}$ & 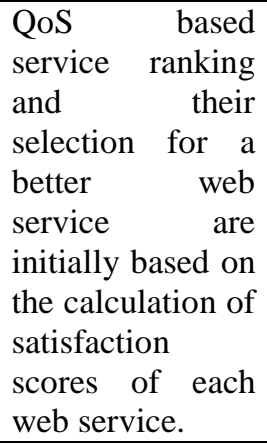 & $\begin{array}{l}\text { Improved by } \\
\text { desegregation } \\
\text { completely } \\
\text { different non } \\
\text { useful properties } \\
\text { into the thought } \\
\text { that helps in } \\
\text { providing higher } \\
\text { results. }\end{array}$ \\
\hline 5 & $\begin{array}{l}\text { Alternating } \\
\text { Least Squares } \\
\text { (ALS) and } \\
\text { Stochastic } \\
\text { Gradient } \\
\text { Descent } \\
\text { (SGD) } \\
\end{array}$ & $\begin{array}{l}\text { A classical } \\
\text { optimization } \\
\text { approach, has } \\
\text { been used for } \\
\text { many other } \\
\text { large-scale } \\
\text { problems }\end{array}$ & $\begin{array}{l}\text { Both ratings and } \\
\text { variables cannot } \\
\text { fit in the memory } \\
\text { of a single } \\
\text { machine }\end{array}$ \\
\hline
\end{tabular}


Table 4.4 Dataset Table

\begin{tabular}{|c|c|c|c|}
\hline $\begin{array}{c}\text { S. } \\
\text { No }\end{array}$ & Algorithm & Dataset & Accuracy \\
\hline 1 & $\begin{array}{c}\text { SRNs } \\
\text { Recommendation } \\
\text { Algorithm } \\
\text { (2011) }\end{array}$ & $\begin{array}{c}\text { Twitter, } \\
\text { Face Book } \\
\text { Dataset }\end{array}$ & $70.23 \%$ \\
\hline 2 & $\begin{array}{c}\text { Dimensionality- } \\
\text { Reduction Algorithm } \\
\text { (2007) }\end{array}$ & $\begin{array}{c}\text { Transactio } \\
\text { n Dataset } \\
\text { (Amazon) } \\
\end{array}$ & $80.23 \%$ \\
\hline 3 & $\begin{array}{c}\text { Response Aware } \\
\text { Probabilistic Matrix } \\
\text { Factorization } \\
(2015)\end{array}$ & $\begin{array}{c}\text { Location } \\
\text { Base } \\
\text { Transactio } \\
\text { n Dataset }\end{array}$ & $78.05 \%$ \\
\hline 4 & $\begin{array}{l}\text { QoS based service } \\
\text { ranking } \\
(2016)\end{array}$ & $\begin{array}{c}\text { Amazon, } \\
\text { Mobile } \\
\text { Transactio } \\
\text { n Dataset }\end{array}$ & $82.22 \%$ \\
\hline 5 & $\begin{array}{l}\text { Alternating Least } \\
\text { Squares (ALS) and } \\
\text { Stochastic Gradient } \\
\text { Descent (SGD) } \\
\text { (2012) }\end{array}$ & $\begin{array}{l}\text { Twitter, } \\
\text { Face Book } \\
\text { Dataset }\end{array}$ & $83.02 \%$ \\
\hline
\end{tabular}

\section{CONCLUSION}

This survey work explores the score forecast and produces LSN in social communication model. This paper presents a completely unique model tagged increased i-SSU, which mixes the similarity matrices ensuing from user-item, user-user and user-location bi-partite graph model likewise. Further, the behaviours of users and user score square measure analyzed and compared.

From a social assets view, it plans to develop parallel techniques in special urban possessions applications, e.g.E-health field, to verify that the advance is universally applicable in varied domains. At nearby, survey scrutiny shows that the i-SSU formula is a lot of sense in predicting score things and likeliness things in LBSN as an outcome of the dramatic growth of on-line social communication model continues, the recommendation in LBSN is widely used everywhere. In the future, from a social property perspective, the started is to develop similar techniques in the E-commerce field, medical field to verify that the approach is universally applicable in varied domains.

\section{REFERENCES}

1. Z. Huang, D.Zeng, and H.Chen,“A comparison of collaborative filtering recommendation algorithms for e-commerce,” IEEE Intelligent Systems, vol. 22, no. 5, pp. 68-78, Sep. 2007.

2. Haiqin. Yang, G. Ling, Y. Su, M. R. Lyu, and I. King, "Boosting response aware model-based collaborative filtering,” IEEE Transactions on Knowledge and Data Engineering, vol. 27, no. 8, pp. 2064-2077, Feb. 2015

3. C. Priya and S. Rani, "Location-aware and personalized collaborative filtering for web service recommendation: a review," International Journal of Computer Applications, vol. 133, no. 14, pp. 1-3, Jan. 2016.

4. H. F. Yu, C. J. Hsieh, S. Si, and I. Dhillon, "Scalable coordinate descent approaches to parallel matrix factorization for recommender systems," in Proc. 12th IEEE International Conference on Data Mining (ICDM), Brussels, Dec. 2012, pp. 765-774.

5. P. Symeonidis, E. Tiakas, and Y. Manolopoulos, "Product recommendation and rating prediction based on multi-modal social networks," in Proc. 5th ACM Conf. Recommender Syst., 2011, pp. 61-68.

6. Fei Hao, Shuai Li, Geyong Min, Hee-Cheol Kim, Stephen S. Yau, “An Efficient Approach to Generating Location-Sensitive Recommendations in Ad-hoc Social Network Environments,” IEEE transactions on services computing, vol. 8, no. 3, may/june 2015.

7. C. Gentile, S. Li and G. Zappella, "Online clustering of bandits,” J.Mach. Learn. Res.,Workshop Conf. Proc., vol. 32, pp. 757-765, 2014.

8. L. Siksnys, J. Thomsen, S. Saltenis, and M. L. Yiu, "Private and flexible proximity detection in mobile social networks," in Proc. Int. Conf. Mobile Data Manage., 2010, pp. 75-84.

9. M. Ye, P. Yin, and W. Lee, "Location recommendation for location-based social networks," in Proc. 18th SIGSPATIAL Int. Conf. Adv. Geographic Inform. Syst., 2010, pp. 458-461.

10. H. Ma, D. Zhou, C. Liu, M. R. Lyu, and I. King, "Recommender systems with social regularization," in Proc. ACM Int. Conf. Web Search Data Mining, 2011, pp. 287-296.

11. Y. Zheng, "Location-based social networks: Users," in Computing with Spatial Trajectories, Y. Zheng and X. Zhou, Eds. New York, NY, USA: Springer, 2011.

12. $\mathrm{P} . \mathrm{Pu}, \mathrm{L}$. Chen, and $\mathrm{R}$. Hu, "A User-centric evaluation framework for recommender systems,” in Proc. ACM 5th ACM Conf. Recommender Syst., 2011, pp. 157164

13. M. Sarwat, J. Bao, A. Eldawy, J. Levandoski, A.Magdy, and F.Mokbel, "Sindbad: A location-based social networking system," in Proc. ACMSIGMODInt. Conf.Manage.Data, 2012, pp. 649-652.

14. Y. R. Lin, J. Sun, H. Sundaram, A. Kelliher, P. Castro, and R. Konuru, "Community discovery via metagraph factorization,” ACM Trans. Knowl. Discovery Data, vol. 5, no. 3, pp. 17-17, 2011.

15. Mehregan Mahdavi, “Designing a recommender system based on Social networks and location based Services," in Proc. 6th ACM Int. Conf. Adv. Mobile Comput. Multimedia, 2008, pp. 163-169.

16. Francesco Ricci, Lior Rokach, Bracha Shapira and Paul B. Kantor, "Recommender Systems Handbook," Springer, ISBN: 978-0-387-85819-7, CSpringer Science + Business Media LLC, 2011. 
E.Kaviyavarshini et al, International Journal of Advanced Research in Computer Science, 9 (3), May-June 2018, 100-104 